\title{
A Simon effect in memory retrieval: Evidence for the response-discrimination account
}

\author{
Peter Wühr \\ Friedrich-Alexander Universität, Erlangen, Germany \\ AND \\ ULRICH ANSORGE \\ Universität Bielefeld, Bielefeld, Germany
}

\begin{abstract}
According to the traditional view, the effects of irrelevant stimulus location on the selection of a spatial response to a nonspatial stimulus feature (Simon effect) result from long-term associations between spatial stimulus codes and spatially corresponding response codes. According to an alternative view, the responsediscrimination account, Simon effects arise from interactions between spatial stimulus codes and response labels in working memory (WM). The latter account predicts Simon effects when participants use spatial labels for response representation in WM, even when the actual responses have no spatial features (e.g., saying the word "plate"). The prediction was tested in an experiment, in which participants first encoded two words at different locations, and then responded to a stimulus by saying the word from the location indicated by stimulus color. The manipulation concerned the correspondence between irrelevant location of the colored stimulus and the retrieval cue for the vocal responses (i.e., word location in the encoding display). A Simon effect in memory retrieval was observed, supporting the response-discrimination account.
\end{abstract}

An important tool for studying human action control and response selection has been to investigate the effects of spatial stimulus-response (S-R) compatibility. These effects arise when stimuli and responses vary on overlapping spatial dimensions (Kornblum, Hasbroucq, \& Osman, 1990). Consider, for example, a task in which participants press a left-side key to a green stimulus and a right-side key to a red stimulus, while each stimulus appears randomly to the left or to the right of fixation. In this task, spatially corresponding conditions (left green $\mathrm{S}$, right red S) produce faster responses and higher accuracy than spatially noncorresponding conditions (left red S, right green S). This finding, called Simon effect, shows that spatial S-R correspondence affects performance even when stimulus location is irrelevant for the task at hand (e.g., Simon \& Rudell, 1967; see Lu \& Proctor, 1995, for a review). The most widely accepted accounts for the Simon effect are dual-route models. These models assume two independent routes through which stimuli might activate responses, the controlled route and the automatic route (e.g., Hommel, 1997; Kornblum et al., 1990; Zhang, Zhang, \& Kornblum, 1999). Through the controlled route, the relevant stimulus feature (e.g., color) activates an arbitrary response to that feature. The controlled route is believed to rest on short-term associations, established in working memory (WM) on the basis of task instructions. In contrast, each spatial stimu- lus feature activates a spatially corresponding response through the automatic route. The automatic route is believed to rest on long-term associations that are independent from the content of WM (e.g., Zhang et al., 1999). In corresponding conditions of the Simon task both routes activate the same response, which is quickly executed. In noncorresponding conditions, however, each route activates a different response, causing response conflict, which delays responding or produces an error.

Some findings, however, raised doubts that locationbased response activation in the Simon task is based on long-term S-R associations. Hommel (1993), for example, found that instructions modulated the Simon effect. In one experiment, participants responded to the color of a visual stimulus by pressing a left or right key, each of which flashed a light on the opposite side. One group of participants was instructed to press keys (e.g., press left key to green stimulus), whereas another group was instructed to flash lights (e.g., flash right light to green stimulus). As expected, the first group produced a regular Simon effect, that is, faster responses with spatial stimulus-key correspondence (i.e., stimulus-light noncorrespondence). In contrast, the second group produced an inverted Simon effect, that is, faster responses with spatial stimulus-light correspondence (i.e., stimulus-key noncorrespondence). These results show that attending to different spatial features of a response modulates the Simon effect.

P.Wühr, peter.wuehr@psy.phil.uni-erlangen.de 
Valle-Inclán and Redondo (1998) reported another challenge to the traditional view. They measured reaction times (RTs) and lateralized readiness potentials (LRPs) in a Simon task. The LRP is an electrophysiological correlate of response activation in primary motor cortex (M1) and supplementary motor area (SMA), indicating whether the left or the right hand is activated more strongly (Coles, 1989). Previous studies had shown that spatially noncorresponding stimulus location can activate the "incorrect" motor cortex in the Simon task; this was taken to indicate automatic response activation on the basis of long-term S-R associations (e.g., Eimer, Hommel, \& Prinz, 1995). Valle-Inclán and Redondo replicated this finding when participants were informed about the relevant S-R mapping before the imperative stimulus was shown. Interestingly, however, they failed to observe "incorrect" response activation in noncorresponding Simon trials when participants received the imperative stimulus before they were informed about the relevant $\mathrm{S}-\mathrm{R}$ mapping. This result suggests that location-based response activation in the Simon task is not automatic. Instead, the Simon effect appears to depend upon the $\mathrm{S}-\mathrm{R}$ mapping being represented in WM.

To account for these results, Ansorge and Wühr (2004) proposed a response-discrimination account of the Simon effect. The central claim is that the Simon effect arises in WM from the interaction of codes representing stimulus location and codes representing response location. Moreover, the account claims that participants can flexibly represent responses in WM (see, also, Hommel, Müsseler, Aschersleben, \& Prinz, 2001), and that Simon effects will only arise if participants use spatial features, or spatial labels, for response representation in WM. According to this view, the Simon effect does not arise from direct interactions between spatial stimulus codes and spatial response (motor) codes, but rather from the interaction between spatial stimulus codes and codes representing responses in WM. Consequently, the response-discrimination account makes two complementary predictions: (1) Simon effects should not arise when spatial responses are represented by (and discriminated on the basis of) nonspatial codes in WM, and (2) Simon effects should arise when nonspatial responses are represented by (and discriminated on the basis of) spatial codes in WM.

Ansorge and Wühr (2004) have tested the first prediction. The idea was that participants most likely discriminate between two spatial responses in a choice-response task (CRT) on the basis of spatial location. In contrast, we hypothesized that participants do not necessarily use spatial features for discriminating between a spatial response and not performing this response in a go/no-go task. Consistent with these assumptions, a group of participants showed a Simon effect in the CRT but not in the go/no-go task when the go/no-go task was performed before the CRT. Interestingly, however, a second group of participants showed Simon effects both in the CRT and in the go/no-go task when the CRT was performed before the go/no-go task. The explanation is that in the latter condition participants transferred their response representation on the basis of location from the CRT to the go/no-go task, which produced Simon effects in the latter task.

The purpose of the present study was to test the second prediction of the response-discrimination account as mentioned above. This prediction states that Simon-like correspondence effects should also arise when nonspatial responses are represented by (and discriminated on the basis of) spatial labels in WM. That is, the participants' use of spatial labels in WM as an internal retrieval cue for a response should suffice for producing a Simon effect, regardless of the to-be-performed response. In the present experiment, participants were presented with two consecutive displays, the encoding and the retrieval display. In the encoding display, two words denoting possible vocal responses were shown, one word to the left and the other one to the right of fixation. The horizontal location of each word changed randomly from trial to trial. Participants had to encode the words together with their respective locations into WM. Then, the retrieval display appeared, in which the color of the square called either for the left word or for the right word from the encoding display. That is, depending upon the color of the square, participants had to vocally report the left word or the right word from the encoding display as quickly as possible. As is usual for a Simon task, the colored square appeared randomly to the left or right of fixation, which produced corresponding and noncorresponding conditions. In corresponding conditions, the location of the square (i.e., the external retrieval cue) matched the internal retrieval cue (i.e., the location of the word in the encoding display) for the vocal response. In contrast, in noncorresponding conditions, the location of the square and the internal retrieval cue for the vocal response did not match.

We expected a Simon effect in WM retrieval, that is, we expected shorter RTs and/or higher accuracy in corresponding than in noncorresponding conditions. Obviously, such effects cannot arise from long-term associations between spatial stimulus codes and spatial response codes because the vocal responses have no spatial features. Instead, such effects must arise from the interaction between codes representing square location in retrieval displays and response-retrieval cues in WM (referring to word location in encoding displays).

A potential problem arises from the fact that participants can use different codes for representing the contents of the encoding display in WM. In particular, participants can either use visual codes (i.e., store a visual image of the encoding display), or they can use verbal codes. The use of a visual image would be problematic because a match between the location of the colored square in the retrieval display, and the location of the to-be-retrieved word in the visual image might also produce congruency effects. ${ }^{1}$ By contrast, the response-discrimination account predicts that the use of spatial labels as retrieval cues for responses in WM should suffice to produce Simon effects, even when verbal codes are used for response representation. Fortunately, the contents of WM are accessible to conscious awareness in humans, and therefore we asked the partici- 
pants after the experiment whether they had used visual or verbal codes for representing the retrieval display in WM. The particular question was whether participants who use verbal codes would show Simon effects in WM retrieval.

\section{METHOD}

\section{Participants}

Twenty-three students (14 female, mean age: 25 years) participated in a single session. The experiment lasted about 30 minutes, and participants received $€ 5$ or course credit. All participants were native German speakers, who classed themselves as having normal (or corrected-to-normal) visual acuity.

\section{Apparatus and Stimuli}

The experiment took place in a dimly lit room. The participants sat in front of a 17-in. color monitor with viewing distance being constrained to $60 \mathrm{~cm}$ by a head-and-chinrest. Participants responded by speaking into a microphone, which triggered a voice key measuring RTs to the nearest millisecond. An IBM-compatible computer controlled the presentation of stimuli and collected vocal RTs. Unless otherwise noted, stimuli were shown in white on a black background. The fixation cross was a plus sign $(10 \times 10 \mathrm{~mm})$. Two different types of displays were used, the encoding display and the retrieval display. The encoding display contained two words, representing the possible vocal responses in the next trial, at different locations. The two responses were randomly drawn from a set of four German words (Kiste [box], Palme [palm tree], Teller [plate], Zettel [docket]). In the encoding display, one word appeared to the left of fixation, and the other one appeared to the right of fixation. A word subtended $25 \times$ $8 \mathrm{~mm}$; the distance between the center of a word and the center of the screen was $50 \mathrm{~mm}$. The retrieval display contained the fixation point at screen center, and a colored square (green or red) to the left or right of fixation. The distance between the center of the square $(15 \times$ $15 \mathrm{~mm}$ ) and the center of the screen was $50 \mathrm{~mm}$.

\section{Procedure}

At the beginning of the experiment, the instructions appeared on the screen and participants could read them at leisure. Participants were instructed to report as quickly as possible the word from the location indicated by the color of the square. Twelve participants were told that a green square referred to the word at the left location, and a red square referred to the word at the right location. Eleven participants received the reverse mapping. Participants were also told that the location of the square varied randomly from trial to trial, and that this was irrelevant for their task. Participants had 48 practice trials before 240 experimental trials.

Each trial contained the following sequence of events. First, the encoding display appeared. Participants could look at the encoding display as long as they wished; they pressed the space key in order to continue. With the keypress, the fixation point appeared at screen center and remained on alone for $500 \mathrm{msec}$. Next the retrieval display appeared for $500 \mathrm{msec}$, followed by a blank screen for $1 \mathrm{sec}$. From the onset of the retrieval display, vocal RTs were measured for $1.5 \mathrm{sec}$. If participants did not respond within this period, a corresponding error message appeared in yellow color for an additional $2 \mathrm{sec}$. No feedback was provided. The experimenter, who sat outside of the laboratory room, monitored the participants' performance online, and recorded each error.

After the experiment, participants rated the relative frequency of using verbal codes or visual imagery for representing the contents of the encoding displays on a five-point scale: (1) only verbal, (2) mostly verbal, (3) sometimes verbal/sometimes visual, (4) mostly visual, and (5) only visual. Instructions for this task distinguished between a verbal encoding strategy ("I rehearsed the words by using inner speech") and a visual encoding strategy ("I encoded a visual image of the display with the words"). Participants could also indicate that they had used a different strategy.

\section{Design}

We varied the spatial correspondence between the location of the to-be-named word in the encoding display and the irrelevant location of the colored square in the retrieval display (corresponding vs. noncorresponding).

\section{RESULTS}

For each participant, we first removed all vocal RTs exceeding two standard deviations from the individual mean. Averaged across participants, $2.9 \%$ of trials with anticipatory responses, and $3.0 \%$ of trials with retarded responses were excluded from further analysis. Two-tailed $t$ tests were used for all comparisons.

The interview revealed that the majority of participants had used verbal codes for memorizing the words from the encoding display: only verbal: 8 , mostly verbal: 7 , sometimes verbal and/sometimes visual: 4, mostly visual: 4, only visual: 0 . No participant reported the use of a third way of representing the encoding displays.

RTs from the complete sample showed a Simon effect in WM retrieval: Spatial correspondence between the irrelevant location of the square in the retrieval display and the location of the to-be-retrieved word in the encoding display produced faster responses than spatial noncorrespondence [701 vs. $740 \mathrm{msec}, t(22)=8.12, p<.001$ ] Error percentages showed no effect $[3.1 \%$ vs. $4.0 \%$, $t(22)=1.28, p=.22]$.

In a next step, we analyzed the results for the subset of participants that had used the verbal encoding strategy in most or all trials $(n=15 ; 9$ female). RTs from these participants showed a significant Simon effect in WM retrieval [689 msec vs. $730 \mathrm{msec}, t(14)=7.09, p<$ $.001]$. Error percentages showed no effect [ $2.6 \%$ vs. $4.2 \%$, $t(14)=1.55, p=.14]$.

Table 1

Response Times (RTs, in Milliseconds) in Corresponding and Noncorresponding Conditions, As a Function of Overall Response Speed (RT Quintile)

\begin{tabular}{|c|c|c|c|c|c|}
\hline & \multicolumn{5}{|c|}{ RT Quintile } \\
\hline & 1 & 2 & 3 & 4 & 5 \\
\hline Corresponding & 514 & 607 & 687 & 773 & 924 \\
\hline Noncorresponding & 567 & 660 & 728 & 805 & 944 \\
\hline Difference & $53^{*}$ & $53^{*}$ & $41^{*}$ & $32 *$ & $20 *$ \\
\hline
\end{tabular}

* Indicates a significant difference (all $p \mathrm{~s}<.01$, two-tailed $t$ test). 
Additional analyses tested for the presence of typical features of the Simon effect. The first analysis investigated whether the size of the Simon effect in WM retrieval decreased when RT increased (e.g., Hommel, 1994). Therefore, RTs in corresponding and noncorresponding conditions were independently rank-ordered, and subdivided into quintiles for each participant. Next, mean RTs from each quintile were entered into a twofactorial ANOVA with correspondence and quintile as within-subjects factors. There were significant main effects of correspondence $[F(1,22)=67.35, p<.001]$ and of quintile $[F(4,88)=326.39, p<.001]$, and a significant two-way interaction $[F(4,88)=12.69, p<.001]$. The interaction indicated a significant decrease of Simon effects with increasing RT (Table 1).

The second analysis investigated whether stimulus-cue correspondence in the immediately preceding trial affected Simon effects in WM retrieval. RTs were entered into a two-factorial ANOVA with preceding correspondence and present correspondence as within-subjects factors. Most importantly, the two-way interaction between preceding correspondence and present correspondence was significant $[F(1,22)=9.52, p<.01]$, indicating larger Simon effects after corresponding $(D=49 \mathrm{msec})$ than after noncorresponding trials $(D=24)$.

\section{DISCUSSION}

The present study showed that the correspondence between the task-irrelevant location of an imperative stimulus and the spatial labels used for response representation in WM produces a pattern of results that resembles the Simon effect. In particular, participants were faster in retrieving and saying a word to the color of a square when the irrelevant location of the square matched the internal retrieval cue for the vocal response in WM than when it did not. The present results suggest, in contrast to the traditional view (e.g., Zhang et al., 1999), that the Simon effect does not depend upon long-term associations between spatial stimulus codes and spatial response codes. Instead, and in accordance with the response-discrimination account (Ansorge \& Wühr, 2004), these results suggest that the Simon effect results from the use of spatial labels for representing (spatial or nonspatial) responses in WM. According to this view, Simon effects do not arise from spatial stimulus codes activating spatial response codes independently from the contents of WM, but rather from spatial stimulus codes activating corresponding retrieval cues for responses in WM. Results of an interview performed after the experiment suggest that the majority of participants had used verbal codes (referring to word location in encoding displays) for representing responses in WM. However, although being suggestive, the results of the interviews are not definitive that participants had exclusively used verbal codes for response representation in WM.

The first point to discuss is whether the present finding actually constitutes a Simon effect. Three facts support this view. First, a recent study has demonstrated that Simon effects can occur in vocal responses (Wühr, 2006).
Second, the Simon effect in WM retrieval decreased when overall RT increased (see Table 1). The observation of larger Simon effects with faster responses is well known from the literature (e.g., Hommel, 1994); it has been explained by the decay of spatial stimulus-code activation. Third, the Simon effect in WM retrieval was larger after corresponding trials than after noncorresponding trials. The observation of sequence effects in our study is particularly compelling evidence for two reasons: (1) In contrast to the traditional task, a study phase intervened between two consecutive trials of the present experiment, which might interfere with the processes producing sequential effects; and (2) the study phase between two consecutive trials increased the temporal interval between trials, which has been shown to reduce the size of sequential effects (Wühr \& Ansorge, 2005). In summary, the similarities between the Simon effect in WM retrieval and the traditional Simon effect suggest that both effects arise from similar mechanisms. However, more work on this issue is clearly desirable.

A secondary finding of the present experiment is that Simon effects do not depend upon the responses having spatial attributes. This observation is consistent with the results of some recent studies by De Houwer and colleagues (De Houwer, 2004; De Houwer, Beckers, Vandoorpe, \& Custers, 2005). In these studies, participants discriminated between centrally presented location words, centrally presented arrows, and between laterally presented color patches with arbitrary vocal responses (e.g.,

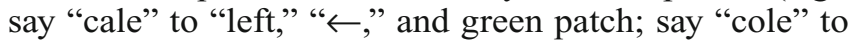
"right," " $\rightarrow$," and red patch). Responses to color patches revealed Simon effects because the congruency between the location of the color patch and the location associated with the vocal response affected performance. This "extrinsic" Simon effect resembles our observation, but we believe that different mechanisms have produced the two effects. The "extrinsic" Simon effect most likely resulted from the compatibility between two concurrently active $\mathrm{S}-\mathrm{R}$ rules. The participants in De Houwer et al.'s (2005) task most likely create a generalized S-R rule (e.g., leftcale) for the whole experiment when at least two different stimuli, referring to spatial location, are consistently mapped onto the same response (De Houwer et al., 2005). In this case, the presentation of a lateralized stimulus activates both the relevant $\mathrm{S}-\mathrm{R}$ rule (e.g., green-cale) and an irrelevant, generalized rule (e.g., left-cale). The compatibility between the two rules affects response selection. In contrast, we believe that the results of the present experiment emerged from the correspondence between irrelevant stimulus location and response labels in WM.

The results of the present study indicate that short-term associations involving spatial information can produce correspondence effects. This conclusion is consistent with the results of studies demonstrating compatibility-frommemory effects (e.g., Hommel, 2002; Tlauka \& McKenna, 1998). For example, Hommel's participants first memorized a display of four stimuli that varied in color, shape, and location. Then, a cue stimulus required to retrieve one of the stimuli on the basis of its color, and to respond to stimulus shape by pressing a left or right key. Interestingly, 
the irrelevant horizontal location of the to-be-retrieved stimulus in the encoding display produced a Simon effect in subsequent responses to the cue, indicating shortterm associations between the different stimulus features. There are important differences between the compatibilityfrom-memory studies and the present study, however. In the former studies, retrieval of a spatial stimulus feature from memory affects the selection of spatial responses to spatially neutral stimuli. In contrast, in the present study, perception of a spatial stimulus feature affects the selection between nonspatial responses, when the selection has to be made on the basis of spatial labels in WM.

A final issue is how the codes representing stimulus location get in touch with the codes representing responses. If the Simon effect does not arise from long-term associations between spatial stimulus codes and spatial response codes, how can spatial stimulus information then activate corresponding responses? On our account, the Simon effect arises from the interaction of codes representing stimulus location and spatial labels used for response representation in WM. Specifically, codes representing stimulus location should tend to activate similar codes (i.e., codes referring to physically or conceptually similar contents) in WM. In the present experiment, the code referring to left square location would have activated the code referring to left word location to a larger extent than the code referring to the right word location, and vice versa. Similarly, in the usual Simon task, the code referring to left stimulus location would activate the code referring to left response location to a larger extent than the code referring to the right response location, and vice versa.

In summary, the present results are incompatible with traditional explanations of the Simon effect, according to which long-term associations between spatial stimulus codes and spatial response codes produce the effect. Instead, the results are consistent with the responsediscrimination account, according to which the interaction between spatial stimulus codes and spatial labels used for response representation in WM produces the Simon effect.

\section{AUTHOR NOTE}

The German Science Foundation (DFG) supported this research through Grant WU 357/2-1 to the first author. We thank Inga Reismann for collecting the data. Moreover, we are grateful to Yang Seok Cho, Bernhard Hommel, and Jan De Houwer for commenting on previous versions of this article. Correspondence concerning this article can be directed to P. Wühr, Friedrich-Alexander Universität, Institut für Psychologie I, Kochstrasse 4, 91054 Erlangen, Germany (e-mail: peter.wuehr@ psy.phil.uni-erlangen.de).

\section{REFERENCES}

ANsorge, U., \& WüHr, P. (2004). A response-discrimination account of the Simon effect. Journal of Experimental Psychology: Human Perception \& Performance, 30, 365-377.

Coles, M. G. (1989). Modern mind-brain reading: Psychophysiology, physiology, and cognition. Psychophysiology, 26, 251-269.

De Houwer, J. (2004). Spatial Simon effects with nonspatial responses. Psychonomic Bulletin \& Review, 11, 49-53.

De Houwer, J., Beckers, T., Vandoorpe, S., \& Custers, R. (2005). Further evidence for the role of mode-independent short-term associations in spatial Simon effects. Perception \& Psychophysics, 67, 659-666.

Eimer, M., Hommel, B., \& Prinz, W. (1995). S-R compatibility and response selection. Acta Psychologica, 90, 301-313.

Hommel, B. (1993). Inverting the Simon effect by intention: Determinants of direction and extent of effects of irrelevant spatial information. Psychological Research, 55, 270-279.

Hommel, B. (1994). Spontaneous decay of response-code activation. Psychological Research, 56, 261-268.

Hommel, B. (1997). Toward an action-concept model of stimulusresponse compatibility. In B. Hommel \& W. Prinz (Eds.), Theoretical issues in stimulus-response compatibility (pp. 281-320). Amsterdam: Elsevier.

Hommel, B. (2002). Responding to object files: Automatic integration of spatial information revealed by stimulus-response compatibility effects. Quarterly Journal of Experimental Psychology, 55A, 567-580.

Hommel, B., Müsseler, J., Aschersleben, G., \& Prinz, W. (2001). The theory of event coding (TEC): A framework for perception and action planning. Behavioral \& Brain Sciences, 24, 849-937.

Kornblum, S., HasbroucQ, T., \& Osman, A. (1990). Dimensional overlap: Cognitive basis for stimulus-response compatibility. A model and taxonomy. Psychological Review, 97, 253-270.

Lu, C. H., \& Proctor, R. W. (1995). The influence of irrelevant location information on performance: A review of the Simon and spatial Stroop effects. Psychonomic Bulletin \& Review, 2, 174-207.

Simon, J. R., \& Rudell, A. P. (1967). Auditory S-R compatibility: The effect of an irrelevant cue on information processing. Journal of Applied Psychology, 51, 300-304.

Tlauka, M., \& McKenna, F. P. (1998). Mental imagery yields stimulus-response compatibility. Acta Psychologica, 98, 67-79.

Valle-Inclán, F., \& Redondo, M. (1998). On the automaticity of ipsilateral response activation in the Simon effect. Psychophysiology, 35, 366-371

WÜHr, P. (2006). The Simon effect in vocal responses. Acta Psychologica, 90, 301-313.

WÜHR, P., \& ANSORGE, U. (2005). Exploring trial-by-trial modulations of the Simon effect. Quarterly Journal of Experimental Psychology, 58A, 705-731.

Zhang, H., Zhang, J., \& Kornblum, S. (1999). A parallel distributed processing model of stimulus-stimulus and stimulus-response compatibility. Cognitive Psychology, 38, 386-432.

\section{NOTE}

1. We thank Jan De Houwer for raising this issue.

(Manuscript received March 6, 2006; revision accepted for publication November 20, 2006.) 